\title{
Analysis of Safety, Medical Resource Utilization, and Treatment Costs by Drug Class for Management of Inflammatory Bowel Disease in the United States Based on Insurance Claims Data
}

\author{
Gráinne H. Long • Amanda R. Tatro • Young S. Oh • Sheila R. Reddy • \\ Ashwin N. Ananthakrishnan
}

Received: April 12, 2019 / Published online: September 27, 2019

(c) The Author(s) 2019

\section{ABSTRACT}

Introduction: Conventional pharmaceutical interventions for inflammatory bowel disease (IBD) provide limited disease/symptom control and are associated with an increased risk of adverse events (AEs). These limitations increase patient morbidity, medical resource utilization (MRU), and costs.

Methods: The IQVIA ${ }^{\mathrm{TM}}$ Real-World Data Adjudicated Claims-US database was leveraged to

Enhanced Digital Features To view enhanced digital features for this article go to https://doi.org/10.6084/ m9.figshare.9772016.

Electronic Supplementary Material The online version of this article (https://doi.org/10.1007/s12325019-01095-1) contains supplementary material, which is available to authorized users.

\section{G. H. Long}

Roche Pharmaceuticals Ltd., Real World Data

Science, Hertfordshire, UK

A. R. Tatro - Y. S. Oh

Genentech, Inc., South San Francisco, CA, USA

S. R. Reddy

Partnership for Health Analytic Research, Beverly

Hills, CA, USA

A. N. Ananthakrishnan $(\bowtie)$

Massachusetts General Hospital, Boston, MA, USA

e-mail: aananthakrishnan@mgh.harvard.edu

A. N. Ananthakrishnan

Harvard Medical School, Boston, MA, USA identify adult patients ( $>18$ years) with Crohn's disease (Crohn's) or ulcerative colitis (UC), who were new and chronic users ( $\geq 60$ days) of oral corticosteroids (OCS), immunosuppressants (IS), anti-tumor necrosis factor agents (antiTNF) or combinations thereof. Using aminosalicylate-treated patients as a reference, we compared AE incidence, MRU, and medical costs across drug classes.

Results: The analysis included 30,676 patients (Crohn's: $n=14,528$; UC: $n=16,148)$. OCS monotherapy was the strongest predictor of any $\mathrm{AE}$ occurring [Crohn's: hazard ratio 1.62 (1.51-1.73); UC: hazard ratio 1.57 (1.49-1.66)]. A similar pattern was observed for severe infection and bone-related conditions. Patients with UC or Crohn's receiving OCS or IS plus OCS were more likely to have emergency department visits, IBD-related hospitalizations/visits/procedures, and gastrointestinal surgery than were patients receiving other therapies. Annualized total medical costs (pharmacy plus hospital service costs) were greatest for anti-TNF plus IS or anti-TNF therapy in both Crohn's and UC. Annualized medical service costs (excluding IBD drug costs) were highest for patients initiating OCS-containing therapies [Crohn's: OCS, $\$ 27,041(24,882-29,200)$ and OCS plus IS, $\$ 23,332(19,889-26,775) ;$ UC: OCS, $\$ 19,659$ $(17,977-21,340)]$.

Conclusion: Although biologic therapies have higher pharmacy costs, treatment decisions should consider the increased AE risks and long- 
term MRU costs associated with chronic use of OCS-containing therapies.

Funding: This study was funded by F. Hoffmann-La Roche Ltd. The journal's Rapid Service Fee and Open Access publication were paid for by ApotheCom on behalf of Genentech, a member of the Roche group who funded the study.

Keywords: Aminosalicylates; Annualized medical cost; Anti-tumor necrosis factor alpha; Claims database; Corticosteroids; Crohn's disease; Gastroenterology; Healthcare resource utilization; Ulcerative colitis

\section{INTRODUCTION}

Inflammatory bowel disease (IBD) is a chronic, relapsing-remitting, inflammatory condition of the gastrointestinal tract [1]. In 2015, it was estimated that 3.1 million adults (1.3\%) in the United States had ever received a diagnosis of IBD [2]. Patients with IBD (i.e., diagnosed with either ulcerative colitis or Crohn's disease) can experience symptoms at a young age [1, 3], necessitating long-term and often costly treatment over their lifetimes [4-6].

Several national and international treatment guidelines for IBD have been published [7-13]. Overall, the recommendations suggest initiating treatment with pharmacologic interventions, such as aminosalicylates, and progressing to corticosteroids and immunosuppressants (IS) $[14,15]$. For patients with moderate to severe symptoms not alleviated by these therapies, guidelines recommend biologic treatments, such as anti-tumor necrosis factor (anti-TNF) agents [9-11, 15]. Notably, the European Crohn's and Colitis Organisation (ECCO) guidelines do not recommend the use of corticosteroids for maintaining remission in IBD $[10,11]$.

With new research informing our understanding of disease pathophysiology and furthering the development of new pharmacologic options, the treatment paradigm for IBD continues to evolve $[10,13,15,16]$. In addition, a broader consensus is emerging which supports the notion that treatment decisions in IBD should move beyond symptom control to encompass longer-term objectives, including endoscopic/histologic healing and reduction in biomarkers of inflammation [17].

While these developments are ongoing, alleviation of symptoms remains an important goal of conventional treatment approaches $[9,10]$. Only one-third of patients with IBD treated using current pharmacologic options experience clinical remission at 1 year [18], and many experience drug-related adverse events (AEs) [18-20]. Therefore, as a result of suboptimal disease control and treatment disutility, patients with IBD can experience significant morbidity and poor quality of life [3-5].

The published literature supports significant medical resource utilization (MRU) and costs associated with IBD management $[4,5,21-25]$, but the extent of this burden has not been fully characterized. To better understand the unmet need among patients with moderate to severe IBD, we evaluated the clinical and economic burden of IBD management in terms of AEs of interest and MRU and associated medical costs using a large real-world US healthcare claims database.

\section{METHODS}

\section{Study Design}

This was a retrospective cohort study using US insurance claims data from the IQVIA ${ }^{\mathrm{TM}}$ RWD Adjudicated Claims-US database [26]. The database includes information about each physician visit, medical procedure, hospitalization, medication dispensed in the outpatient setting and the date of service/prescription, number of days of medication supplied, and tests performed. Healthcare costs (claims paid) were reported as fee-for-service equivalents. The study included patients with information during the identification period July 1, 2011, to June 30, 2014) with a 1-year pre-index/baseline period (see Fig. 1; Supplemental Digital Content 1 ). The 1-year pre-index period (referred to here as baseline) before the first qualifying IBD drug claim allowed clear determination that the first 

a

\section{Treatment cohort}

Patients who received treatments of interest $\mathrm{n}=\mathbf{3 7 , 1 0 5}$
Identification

Patients with $\geq 2$ inpatient or non-diagnostic outpatient claims for Crohn's disease at least 7 days apart within the study period $n=143,971$

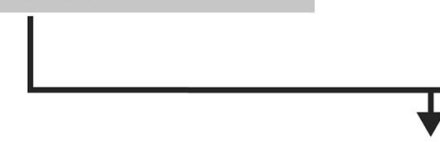

Screening

Combined treatment of interest and reference group $n=47,085$

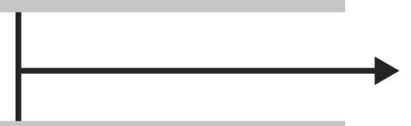

Excluded patients ${ }^{\mathrm{a}}$ $\mathrm{n}=32,557$

\section{Reference group}

Patients who received aminosalicylate $n=9,980$

b

\section{Identification}

Patients with $\geq 2$ inpatient or non-diagnostic outpatient claims for ulcerative colitis at least 7 days apart within the study period

$$
n=143,700
$$

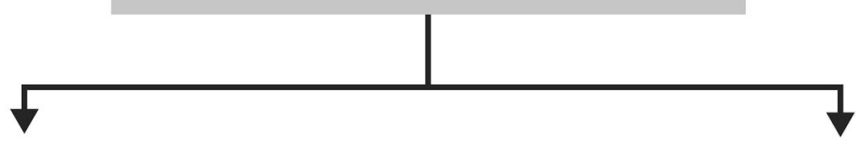

Treatment cohort

Patients who received treatments of interest

$$
n=22,311
$$

opulation included in analysis

$\mathbf{N}=\mathbf{1 4 , 5 2 8}$

(includes 16,981 treatment episodes)

Reference group

Patients who received aminosalicylate

$$
\mathrm{n}=\mathbf{2 1 , 7 4 6}
$$

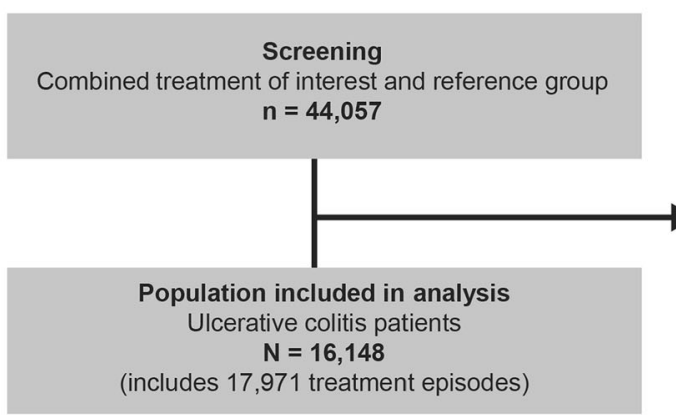

Excluded patients ${ }^{\mathrm{a}}$ $n=27,909$ 
4Fig. 1 Patients included in each disease cohort. a Crohn's disease. b Ulcerative colitis. ${ }^{a}$ Reasons for exclusion: age $\geq 18$ years at index date; chronic IBD treatmentrelated $\mathrm{AE}$ prior to the index date; break to enrollment year prior to the index date/60 days of follow-up; evidence of an alternative indication for anti-TNF therapy during the study period. $A E$ adverse event, $I B D$ inflammatory bowel disease, $T N F$ tumor necrosis factor

exposure to an IBD agent represented a new or inception treatment.

\section{Patients}

Eligible patients consisted of those who, during the identification period, were aged $>18$ years, had $\geq 2$ medical claims (inpatient or non-diagnostic outpatient $\geq 7$ days apart), and were given a diagnosis of ulcerative colitis (ICD-9CM: 556.x) or Crohn's disease (ICD-9-CM: 555.x), with $>1$ qualifying diagnosis (claim) in the year preceding treatment index date (baseline period). In addition, patients were required to be new users (i.e., they were required to have a new claim for an IBD treatment of interest during the identification period to minimize risk of misclassification of exposure). Patients with Crohn's disease with $\geq 2$ claims of UC and UC patients with $\geq 2$ claims of Crohn's disease were excluded. Treatments of interest included chronic ( $\geq 60$ days) [26] oral corticosteroids (OCS), IS, an anti-TNF or combinations thereof (see Table 1; Supplemental Digital Content 2 for full list). The identification period used in this analysis-July 2011-2014-preceded the licensing of vedolizumab in the United States, and other more recently approved therapies; therefore, these agents are not included in these analyses.

Patients with specific conditions for which biologic or IS therapy could be prescribed or any condition that could complicate treatment assessment or confound the evaluation of AEs of interest were excluded (See "Methods" Section I, Supplemental Digital Content 3 for full details of eligibility criteria). For example, patients who had evidence of an alternative indication requiring aTNF therapy were excluded [e.g., plaque psoriasis $(696.1 \mathrm{x})$, psoriatic arthritis (696.0x), rheumatoid arthritis (714.0x), ankylosing spondylitis (720.0x)] were excluded. In addition, patients who had one or more inpatient or outpatient non-diagnostic claim of a chronic IBD treatment-related AE [type 2 diabetes (250.x0, 250.x2), cataract (366.xx, E932.0, CPT 66982, 66983, 66984), glaucoma $(365.1 \mathrm{x}-365.9 \mathrm{x})$, osteoporosis (733.0x), congestive heart failure (428.xx), and venous thromboembolism (415.xx, 451.xx, 453.xx)] prior to the index date were also excluded.

\section{Treatments and Treatment- Attributable At-Risk Period}

This was an incident cohort design. Definitions for treatment duration, treatment gaps, and end of therapy were based on claims data (See "Methods" Section II, Supplemental Digital Content 4 for additional details on treatment duration and follow-up period). The index date was the first use of each therapy of interest in the identification period. If 2 medications were dispensed within 30 days of each other and overlapping for at least 60 days, this was classified as combination therapy [27]. Patients who changed treatments during the study identification period could be included in multiple treatment groups if they met the selection criteria.

Patients could not have had a claim for the index therapy during the baseline period (1-year pre-index period). Patients with continuous use of corticosteroids of at least 60 days during the baseline period were excluded. Notably, in the case of corticosteroids, acute exposure (i.e., $<60$ days of use) was allowed during the baseline period. Continuous use of drugs for at least 60 days was defined as chronic use [26]. To evaluate the association between treatments and certain AEs of interest (see Tables 2, 3, Supplemental Digital Content 5 for ICD codes related to AEs of interest and their resolution), a minimum exposure to the index treatment of 60 days after the index date was required. To capture all treatment-related AEs, the follow-up period included the time up to 60 days after the end of treatment or until the end of enrollment, 
the end of the study, or a treatment switch, whichever occurred first. Switching within a drug class was considered continuous treatment within the class, and data were not analyzed at the individual agent level.

\section{Aminosalicylate Reference Group}

The reference group comprised patients taking only aminosalicylates, with no record of other treatments of interest. To reduce the probability of capturing only patients who were recently given an IBD diagnosis and who were less sick, aminosalicylate users were not required to be new users; rather, they were selected based on a random aminosalicylate fill date in the identification period.

\section{Outcomes of Interest}

The primary outcomes of interest included $5 \mathrm{AE}$ outcomes (any AE, severe infections [20], bonerelated conditions $[28,29]$, arthralgia, and serious hepatic events) and 6 types of healthcare resource utilization (HCRU) [any inpatient hospitalization, any emergency department (ED) visit, any IBD-related hospitalization, any IBD-related gastrointestinal (GI) surgery, any IBD-related ED visit, and any IBD-related procedure (see Tables 4, 5, Supplemental Digital Content 6 for IBD-related procedures and endoscopy codes)], along with 2 annualized costs (all-cause healthcare costs by treatment, and all-cause healthcare costs by AEs) [21]. A more detailed list of the AEs monitored (including selected malignancies $[20,28,29]$ and cardiovascular events) [29] and additional details on HCRU outcomes are included in the Supplementary Methods (see Methods Section III, Supplemental Digital Content 7 for additional details of study outcomes). Diseaserelated and overall HCRU and costs were assessed and included the cost of pharmacologic interventions and inpatient and outpatient services expressed on an annualized basis [21].

\section{Baseline Measures}

Recorded baseline data included demographic characteristics, Charlson Comorbidity Index score (this index predicts the 1-year mortality based on 22 comorbid conditions such as heart disease) [30], number of inpatient admissions, proportion of patients with $\geq 1$ ED visit, proportion of patients with $\geq 1$ IBD-related hospitalization, comorbidities, tobacco use, disease severity [31], behavior parameters, and all medications (see also Methods Section IV and Table 6, Supplemental Digital Content 8 for additional details on baseline measures). Disease severity was expressed as a risk score (range 0-6) that was calculated as a sum of points for individual patients based on the following point assignments, 0 or 1 point each for the absence or presence, respectively, of each of the following at baseline: anemia, requirement for blood transfusion, malnutrition, total parenteral nutrition, occurrence of Clostridioides difficile infection, and occurrence of IBD-related inpatient hospitalization [31].

\section{Statistical Analysis}

All analyses were conducted separately for Crohn's disease and ulcerative colitis patients. Descriptive statistics were reported and included mean and standard deviation (SD) for continuous variables and patient count and percentages for categorical variables, stratified by the treatment groups of interest. Univariate comparisons included statistical tests of significance $\left(\chi^{2}, F\right.$ test, or Kruskal-Wallis rank-sum test). Rates of AEs were reported in units of perpatient-years to account for the variable time that patients are at risk for the event. Healthcare utilization outcomes were annualized to account for variable follow-up time [21]. Multivariate analyses were based on Cox proportional hazards regression, negative binomial regression, logistic regression, or linear regression analysis depending on outcome variable distribution and adjusted by significant sociodemographic, clinical, and disease severity covariates as outlined below. 
Two sets of covariates were initially included in all models: (1) a priori: age, sex, region, and proportion of days covered (defined as ratio of total days of supply of index medication dispensed divided by days between the index date and end of follow-up); (2) other: Charlson comorbidity index [30], baseline number of prescriptions, baseline risk score, tobacco use, and Crohn's disease behavior (for Crohn's disease patients only) [31]. The final models included all a priori covariates and significant "other" covariates (significance level to drop out a covariate was $\geq 0.05$ ). Based on previous publications [32, 33], which proposed a minimum of 10 events per predictor variable, the categories of anti-TNF plus IS, anti-TNF plus OCS, and anti-TNF plus IS plus OCS were combined into 1 group (anti-TNF combined) to ensure sufficient outcome events for robust multivariate analyses; in addition, multivariate analyses were only carried out for those outcome events occurring in sufficient numbers.

For IBD treatment-related AEs, Cox regression models were used. The proportional hazards assumption was checked for all covariates, and a time-dependent variable (interaction term of covariate with log of time) was included if the proportional hazards assumption was violated. For utilization outcomes, negative binomial regression was conducted to model the event rate (number of events per year of exposure) with the use of offset (log of exposure). A linear regression model with repeated measurement adjustment was used for annualized cost outcomes to account for the correlation between multiple treatment episodes for the same patient. As a sensitivity analysis, the cost models were weighted with the duration of follow-up.

All data transformations and statistical analyses were performed using SAS, v.9.4 (SAS Institute, Cary, NC, USA). Tests performed were 2-sided, with a significance level of 0.05 . No corrections for multiple comparisons were applied.

\section{Ethical Considerations}

This article is based on real world data and does not contain any studies with human or animal subjects performed by any of the authors. All data analyzed were de-identified, as required under the US Health Insurance Portability and Accountability Act privacy rule. The dataset for this study contained no protected health information, and the use of the data was determined to be exempt from ethical approval.

\section{RESULTS}

\section{Patient Population}

This analysis included 30,676 unique patients (Crohn's disease: $n=14,528$; ulcerative colitis: $n=16,148$ ) with 34,952 unique treatment episodes (Crohn's disease: $n=16,981$ and ulcerative colitis: $n=17,971$ ) (Fig. 1 ). Thus, $16.9 \%$ and $11.3 \%$ of treatment episodes in the Crohn's disease and ulcerative colitis groups, respectively, represent a change in treatment by a patient during the study period. Baseline demographics and disease characteristics are reported in Table 1. Disease was slightly more severe at baseline in patients with Crohn's disease [mean disease severity risk score (SD) 0.44 [0.74)] than in patients with ulcerative colitis [0.28 (0.65)], and the rate of tobacco use was higher $(11.6 \%$ vs. $4.7 \%)$. Consistent with the observation of increased disease severity risk score, patients with Crohn's disease were almost twice as likely to have had previous inpatient hospitalization $(25.3 \%)$ as ulcerative colitis patients $(14.8 \%)$, and there were more episodes of anti-TNF treatment (monotherapy or combinations) in the Crohn's disease cohort $(39.5 \%)$ than in the ulcerative colitis cohort $(15.9 \%)$. Correspondingly, there were more episodes of less intensive intervention with aminosalicylates in the ulcerative colitis cohort (44.3\%) compared with the Crohn's disease cohort (20.6\%). The most common (occurring in $>10 \%$ of patients) IBD-related comorbidities in Crohn's disease and ulcerative colitis patients were hypertension $(20.3 \%$ and $22.1 \%$, respectively) and hyperlipidemia (12.3\% and $16.9 \%$, respectively). 
Table 1 Baseline demographics and disease characteristics

\begin{tabular}{|c|c|c|}
\hline & Crohn's disease & Ulcerative colitis \\
\hline Treatment episodes ${ }^{\mathrm{a}},(n)$ & 16,981 & 17,971 \\
\hline Unique patients ${ }^{\mathrm{a}},(n)$ & 14,528 & 16,148 \\
\hline Age, $[$ mean $(S D)$, year $]$ & $42.2(14.8)$ & $45.4(14.4)$ \\
\hline Female, $[n(\%)]$ & $8860(52.2)$ & $8301(46.2)$ \\
\hline Charlson comorbidity index ${ }^{\mathrm{b}}$, mean (SD) & $1.0(1.6)$ & $1.2(1.7)$ \\
\hline$<2, n(\%)$ & $12,438(73.2)$ & $12,157(67.6)$ \\
\hline$\geq 2, n(\%)$ & $4543(26.8)$ & $5814(32.4)$ \\
\hline Baseline risk score $[31](0-6)^{c}$, mean $(S D)$ & $0.44(0.74)$ & $0.28(0.65)$ \\
\hline$<2, n(\%)$ & $15,557(91.6)$ & $16,937(94.2)$ \\
\hline$\geq 2, n(\%)$ & $1424(8.4)$ & $1034(5.8)$ \\
\hline Number of prescription fills, mean (SD) & $25.4(24.3)$ & $23.7(21.2)$ \\
\hline Tobacco use ${ }^{\mathrm{d}}, n(\%)$ & $1966(11.6)$ & $850(4.7)$ \\
\hline \multicolumn{3}{|l|}{ Disease behavior, $n$ (\%) } \\
\hline Fistulizing & $609(3.6)$ & - \\
\hline Obstructing & $2063(12.1)$ & - \\
\hline Fistulizing and obstructing & $509(3.0)$ & - \\
\hline Inflammatory & $13,800(81.3)$ & - \\
\hline Any inpatient hospitalizations, $n(\%)$ & $4302(25.3)$ & $2656(14.8)$ \\
\hline \multicolumn{3}{|l|}{ IBD-related comorbidities ${ }^{\mathrm{e}}, n(\%)$} \\
\hline Cardiovascular disease & $379(2.2)$ & $449(2.5)$ \\
\hline COPD/asthma & $1414(8.3)$ & $1169(6.5)$ \\
\hline Hyperlipidemia & $2082(12.3)$ & $3035(16.9)$ \\
\hline Liver disease & $77(0.5)$ & $82(0.5)$ \\
\hline Renal disease & $296(1.7)$ & $257(1.4)$ \\
\hline Hypertension & $3442(20.3)$ & $3970(22.1)$ \\
\hline Time on treatment (proportion days covered), mean (IQR) & $0.923(0.793-0.993)$ & $0.938(0.804-1.000)$ \\
\hline \multicolumn{3}{|l|}{ Treatments of interest, $n(\%)$} \\
\hline OCS & $3352(19.7)$ & $4126(23.0)$ \\
\hline IS & $2351(13.8)$ & $1935(10.8)$ \\
\hline Anti-TNF & $3947(23.2)$ & $1612(9.0)$ \\
\hline IS + OCS & $1074(6.3)$ & $1093(6.1)$ \\
\hline Anti-TNF + IS & $1409(8.3)$ & $567(3.2)$ \\
\hline Anti-TNF + OCS & $982(5.8)$ & $485(2.7)$ \\
\hline
\end{tabular}


Table 1 continued

\begin{tabular}{llc}
\hline & Crohn's disease & Ulcerative colitis \\
\hline Anti-TNF + IS + OCS & $373(2.2)$ & $187(1.0)$ \\
Aminosalicylate & $3493(20.6)$ & $7966(44.3)$ \\
\hline
\end{tabular}

Patients with Crohn's disease and ulcerative colitis identified in the IQVIA ${ }^{\mathrm{TM}}$ Real-World Data Adjudicated Claims-US database (2010-2015)

$T N F$ tumor necrosis factor, COPD chronic obstructive pulmonary disease, IBD inflammatory bowel disease, IQR interquartile range, $I S$ immunosuppressant, $O C S$ oral corticosteroid, $S D$ standard deviation

a All analyses were conducted per treatment episode because unique patients could be identified in more than 1 treatment group

${ }^{\mathrm{b}}$ A measure of overall level of illness validated for use in insurance claims data based on ICD-9 diagnosis codes for the following comorbidities: myocardial infarction, congestive heart failure, peripheral vascular disease, cerebrovascular disease, dementia, chronic pulmonary disease, rheumatic disease, peptic ulcer disease, mild liver disease, diabetes without complication, diabetes with complication, hemiplegia or paraplegia, renal disease, any malignancy, moderate or severe liver disease, metastatic solid tumor, AIDS/HIV. A higher score indicates more chronic conditions

c Baseline risk score (range 0-6); 1 point each for anemia, requirement for blood transfusion, malnutrition, total parenteral nutrition, occurrence of Clostridioides difficile infection, and occurrence of IBD-related inpatient hospitalization. The risk score is the sum of the total points

d Any diagnosis of tobacco use [ICD-9-CM discharge code: 305.1 (tobacco abuse disorder), v15.82 [history of tobacco use)] e IBD-related defined as having an ICD-9-CM diagnosis code of Crohn's disease or ulcerative colitis in the primary diagnosis field

\section{Adverse Events of Interest}

Among patients with Crohn's disease, the rate of any AE (as defined in "Methods") was 12,552 per 10,000 patient-years (TTPY); the rate was similar for patients with ulcerative colitis $(11,700 /$ TTPY). The most common AEs among patients with Crohn's disease included bonerelated conditions (8039/TTPY), serious infections (1740/TTPY), and arthralgia (1597/TTPY). These AEs were also the most common in the ulcerative colitis cohort: bone-related conditions (7254/TTPY), serious infections (1246/ TTPY), and arthralgia (1614/TTPY).

Adjusted Cox regression models found OCS monotherapy and combination therapy of OCS and IS to be the strongest predictors of the occurrence of any AE in patients with Crohn's disease [hazard ratio (HR) $1.62 \quad(95 \%$ CI 1.51-1.73) and HR 1.46 (95\% CI 1.32-1.61), respectively) or ulcerative colitis [HR 1.51 (95\% CI 1.49-1.66) and HR 1.36; 95\% CI 1.23-1.49), respectively] (Table 2). OCS monotherapy and combination therapy of OCS and IS were also the strongest predictors of severe hepatic events and bone-related conditions in patients with Crohn's disease or ulcerative colitis (Table 2). Both OCS and anti-TNF-containing regimens were the strongest predictors of severe infection in patients with Crohn's disease and ulcerative colitis (Table 2). The risk for arthralgia was increased with anti-TNF-containing regimens compared with aminosalicylates and was highest in ulcerative colitis patients treated with anti-TNF and IS (HR 1.56; 95\% CI 1.31-1.87).

\section{HCRU and Costs}

Patients with Crohn's disease or ulcerative colitis receiving OCS or IS plus OCS were more likely to require ED visits, IBD-related hospitalization, doctor visits or procedures, and GI surgery than patients receiving other therapies (Table 3). Within the Crohn's disease cohort, the annual rates of GI surgery and other IBD-related procedures or events were approximately threefold higher for OCS (0.198 and 0.927 events/year, respectively) OCS-containing regimens $(0.238$ 


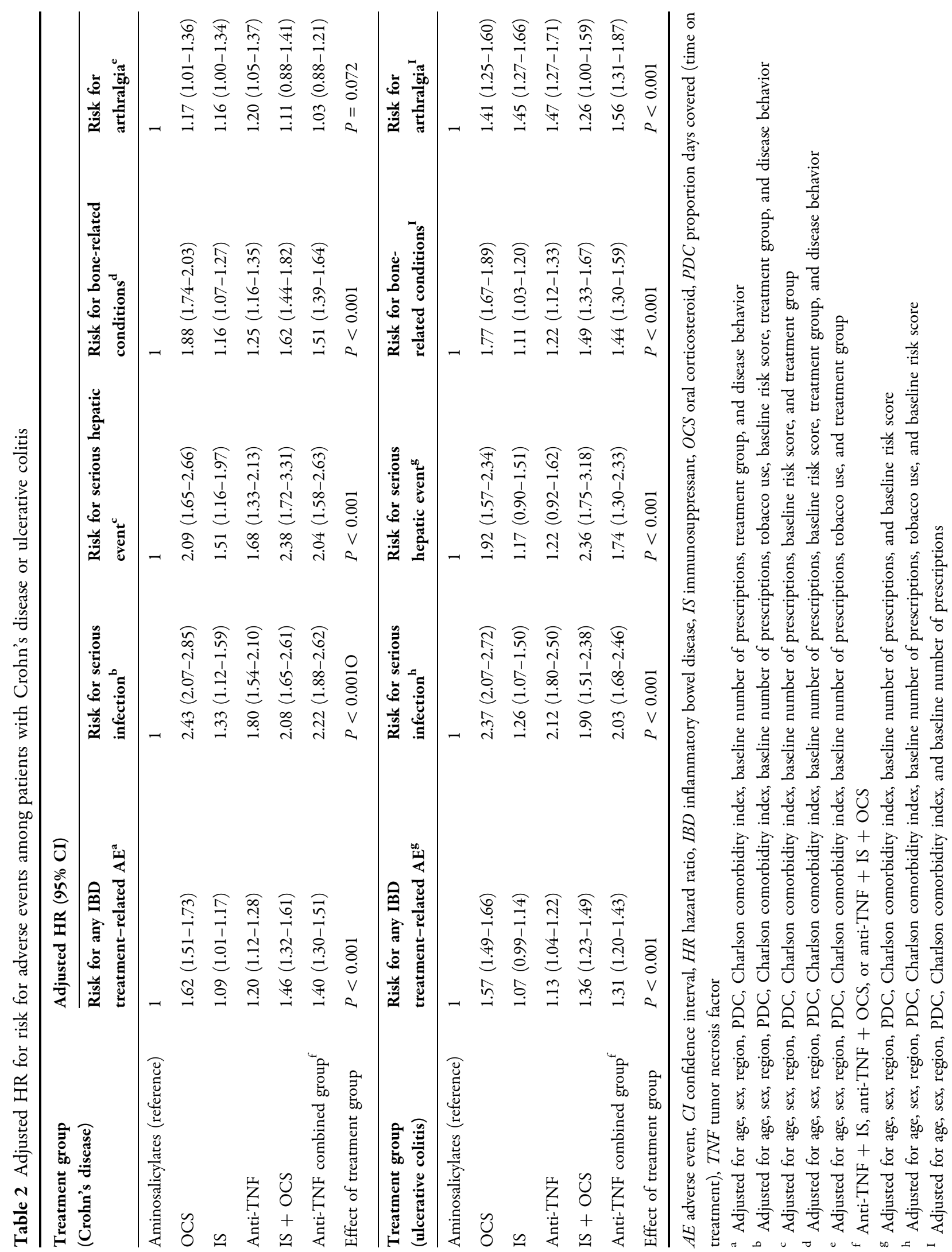


and 0.841 events/year, respectively) than for anti-TNF (0.074 and 0.383 events/year, respectively), and were approximately 1.5 to twofold higher than in the anti-TNF combined group (0.135 and 0.623 events/year, respectively). Similar trends were observed in the ulcerative colitis cohort in the comparison of OCS-containing regimens with anti-TNF; however, the rates of GI surgery, IBD-related hospitalization, and IBD-related ED visits were similar between the OCS-containing regimens and the anti-TNF combined group, with the only notable exception being the increase observed in the IBD-related procedures category (1.020 for OCS vs. 0.721 for anti-TNF combined group) (Table 3).

Annualized total medical costs were greatest for anti-TNF plus IS or anti-TNF therapy for Crohn's disease and ulcerative colitis (Table 4). However, consistent with the data observed in the analyses of HCRU, the annualized medical service costs (excluding IBD drug costs) were highest for patients initiating OCS-containing therapies (Crohn's disease: OCS, US\$27,041, and OCS plus IS, \$23,332; ulcerative colitis: OCS, $\$ 19,659)$ followed by other index therapies [Crohn's disease: aminosalicylates, $\$ 10,823$, and anti-TNF plus IS, $\$ 19,151$ $(P<0.001)$; ulcerative colitis: aminosalicylates, $\$ 7980$, and anti-TNF plus IS, $\$ 18,771$ $(P<0.001)]$.

\section{DISCUSSION}

In this report, we leveraged a large US adjudicated healthcare claims database to characterize the clinical and economic burden of IBD treatment limitations in terms of AEs of interest, HCRU, and associated medical costs. The analyses considered and adjusted for several demographic and disease covariates including baseline disease severity risk score [31].

Patients with IBD receiving chronic treatment ( $\geq 60$ days) with OCS or OCS-containing agents experienced an increased risk for severe infection, bone conditions, and serious hepatic events compared with those receiving other therapies. This is consistent with previous findings that long-term use of corticosteroids may be associated with serious side effects [34].
In addition, patients with IBD receiving OCS or OCS-containing agents also required more healthcare resources, including several IBD-related healthcare resources. These data are reflective of both the increased relative risk of AEs in patients receiving OCS or OCS-containing regimens compared with other index treatments and the limited control of the underlying IBD.

As expected, total medical costs were greatest for anti-TNF therapies in both disease cohorts. This is likely because of the high cost of biologics, although results of previous studies and the current analyses suggest that the high cost of these drugs may be compensated for by a reduction in surgical and hospitalization rates [35], although further research explicitly examining this question is needed. Notably, the current study was conducted prior to the advent of biosimilars; thus, the current and future costeffectiveness of biologics may potentially be more favorable.

Alongside an increased AE risk, OCS regimens were associated with higher rates of HCRU than were other therapies. Indeed, when drug costs were removed from consideration, medical service costs were highest for OCS use in both the Crohn's disease and the ulcerative colitis cohorts. In addition, patients receiving OCS and OCS-containing regimens had greater need for IBD-related procedures and events. With regard to arthralgia, the study design did not account for the occurrence of this event prior to the initiation of the index treatment. As arthralgia is a common extra-intestinal symptom in patients with IBD [36, 37], the increased incidence of this event in patients receiving anti-TNF therapies may be a reflection of the manifestation of more severe disease and the limitation of the intervention to ameliorate the condition.

Taken together, these data highlight the importance of considering the downstream benefits of alternate therapy options in the treatment of IBD. A systematic review of cost-utility analyses comparing biologics with conventional treatment and other interventions for IBD, suggests that biologics are costeffective for the induction treatment of active and severe IBD, especially when considering 


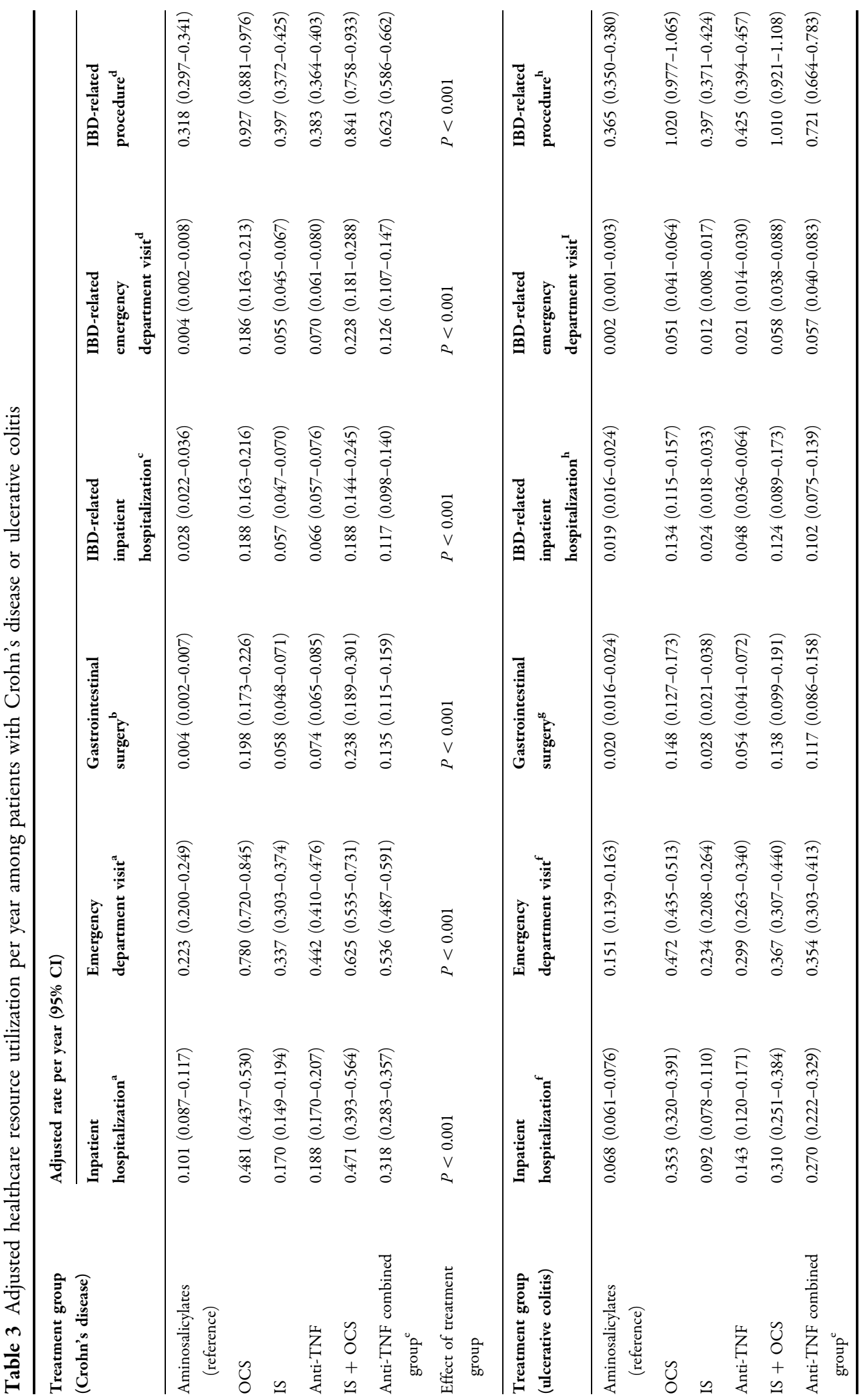




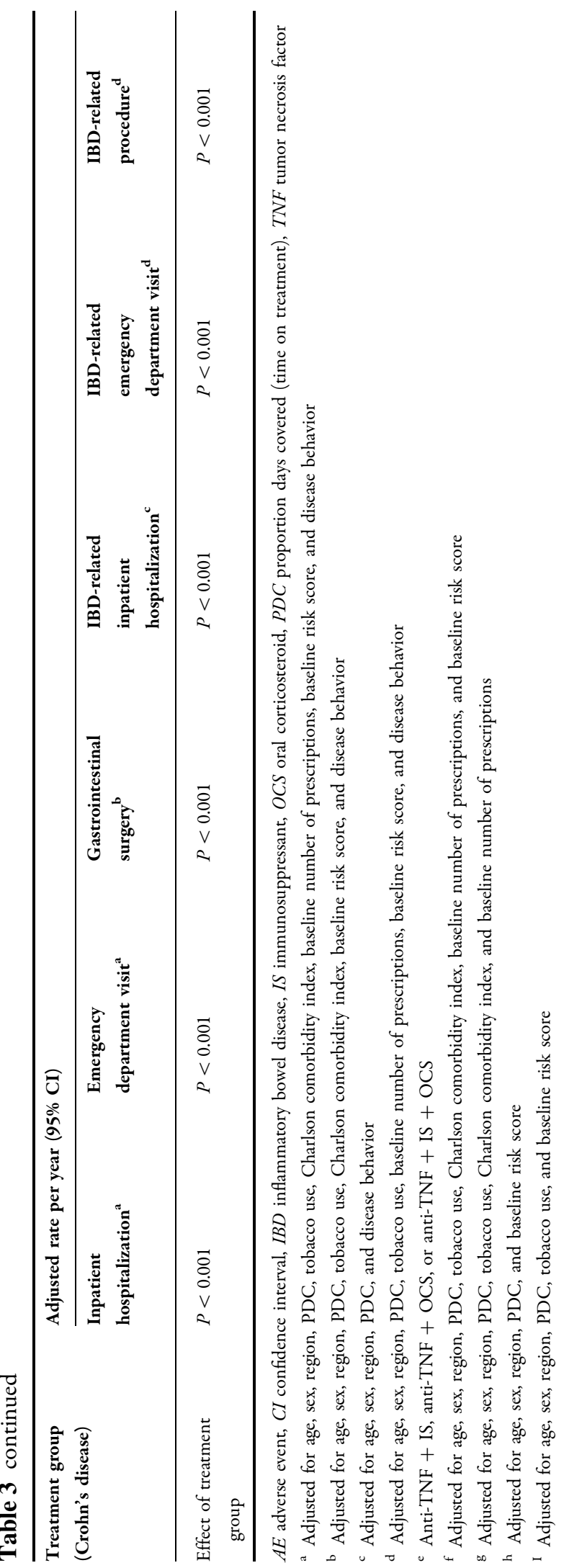

both direct and indirect costs [38]. Given the reduced risk of AEs and requirement for healthcare resource utilization, biologics are potentially associated with better patient quality of life, although we could not address this due to lack of quality of life data in this study. As drug development continues, and newer, safer therapies reach the market, initial drug cost versus subsequent HCRU and patient satisfaction is likely to become an increasingly important factor of therapeutic decision-making for personalized IBD treatment regimens.

The study had several limitations. First, it used a database of adult patients who were commercially insured, which might limit the generalizability of the analysis to a pediatric population, or to uninsured individuals or those with other types of insurance. Second, the use of the aminosalicylate treatment group as a reference may not have been optimal as it likely included patients with less severe disease compared with the patients requiring biologics or IS. Third, although we used multivariate analysis adjusting for key demographic and clinical differences between groups, including a disease severity proxy based on the presence of various clinical conditions and hospitalization, we did not control for unobserved differences between groups which may have been residual source of selection bias. Fourth, we did not adjust for patients who were included in more than one treatment group, as may have been the case for patients who received different treatments at different times during the study period. Fifth, we did not adjust for multiple comparisons. Sixth, the date of diagnosis was not available for all individuals in the database, and, therefore, the interval between diagnosis and initiation of treatment could not be evaluated. Finally, as may be expected in an insurance claims database analyses, there was potential for errors in coding and disease classification, in establishing timing based on claims data, and in the acquisition of comorbidity and demographics information. Nonetheless, the IQVIA $^{\text {TM }}$ RWD Adjudicated Claims-US database provided a large, anonymous dataset that is not based on self-report and is representative of the commercially insured adult US population with IBD. 


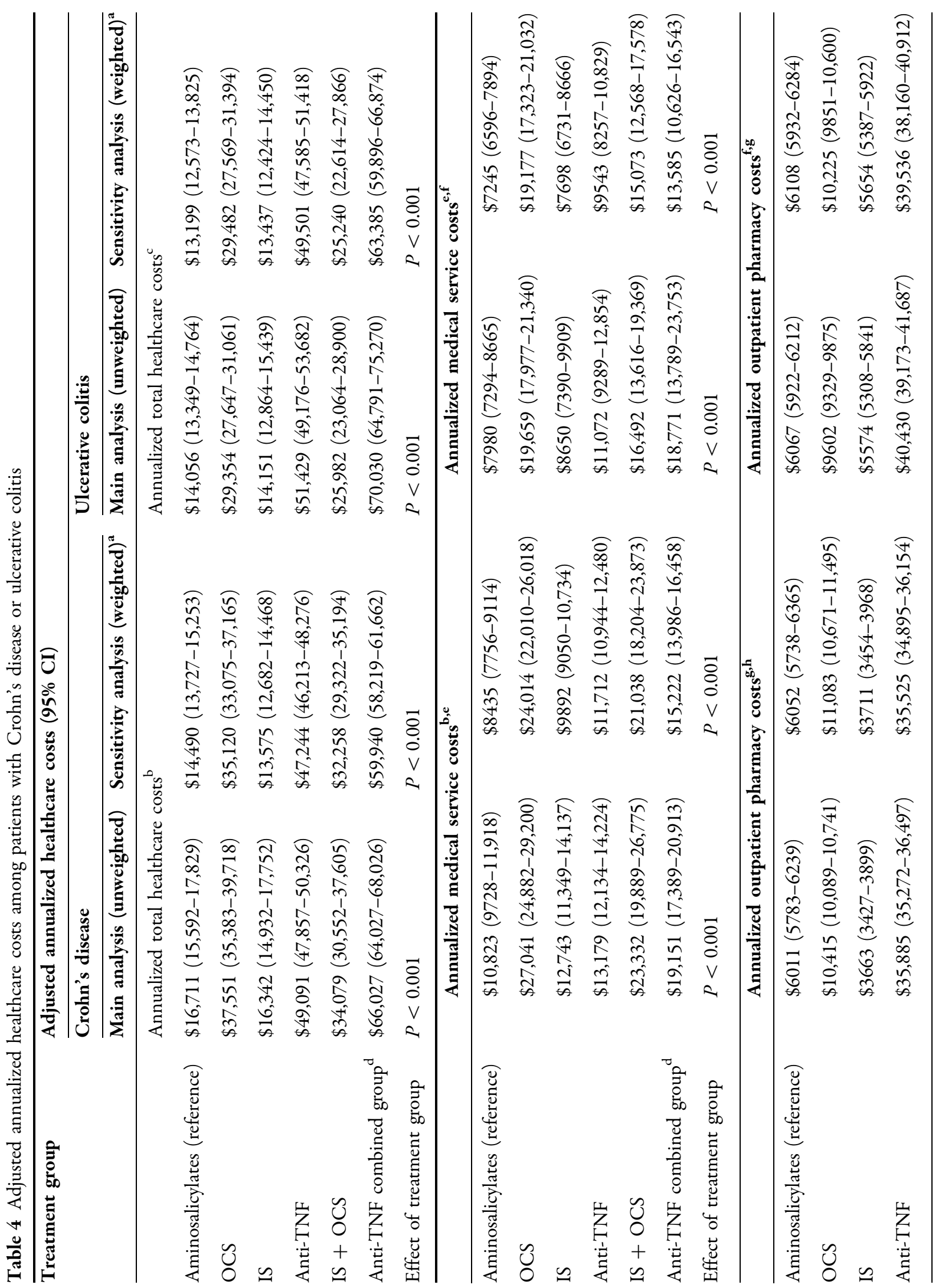




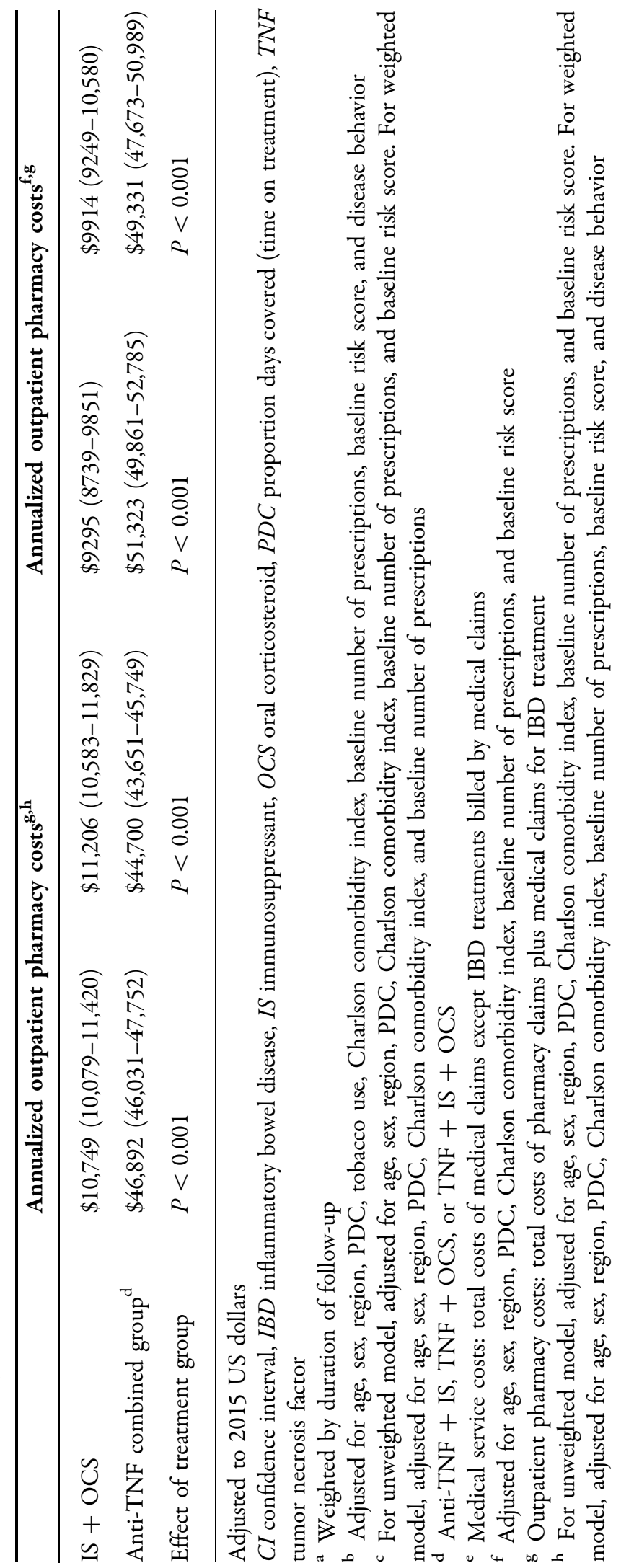




\section{CONCLUSION}

This claims-based analysis revealed the limitations of existing treatments for IBD, particularly of OCS and OCS-containing agents. These regimens are associated with a notable clinical and/ or economic burden because of their unfavorable safety profile and limited effectiveness in controlling IBD symptoms and preventing disease progression compared with biologics (antiTNFs). Accordingly, clinicians should consider initial drug costs in the context of downstream medical resource utilization, overall cost, and the long-term benefit to patients.

\section{ACKNOWLEDGEMENTS}

Funding. This study was funded by F. Hoffmann-La Roche Ltd. The journal's Rapid Service Fee and Open Access publication were paid for by ApotheCom on behalf of Genentech, a member of the Roche group who funded the study.

Medical Writing and Editorial Assistance. Writing and editorial assistance for this manuscript was provided by Sally Mitchell, $\mathrm{PhD}$, and Jerome Sah, PhD, of ApotheCom, Yardley, PA. Support for this assistance was funded by F. Hoffmann-La Roche Ltd.

Authorship. All named authors meet the International Committee of Medical Journal Editors (ICMJE) criteria for authorship for this article, take responsibility for the integrity of the work as a whole, and have given their approval for this version to be published.

Prior Presentation. The analysis and data reported in this manuscript were previously presented at the following congresses: Long $G$ et al. P1058. Presented at the 25th United European Gastroenterology Week; October 28-November 1, 2017; Barcelona, Spain. Long G et al. P-002. Presented at the 2017 Advances in Inflammatory Bowel Diseases; November 9-11, 2017; Orlando, Florida.
Disclosures. Gráinne H. Long was an employee of Roche Pharmaceuticals Ltd. at the time of manuscript development and is currently an employee of AstraZeneca. Amanda R. Tatro was an employee of Genentech, Inc., a member of the Roche group at the time of the writing of manuscript development and owns shares of Roche stock. Amanda R Tatro is currently an employee of Roche. Young S. Oh is an employee of Genentech, Inc., a member of the Roche group, and owns shares of Roche stock. Sheila R. Reddy is an employee of Partnership for Health Analytic Research, LLC, a health services research company hired by Genentech, Inc. and Roche Pharmaceuticals Ltd. to conduct this research. Ashwin Ananthakrishnan reports participating in an advisory board with Takeda and receiving grants from Pfizer during the conduct of the study.

Compliance with Ethics Guidelines. This article is based on previously conducted studies and does not contain any studies with human or animal subjects performed by any of the authors. All data analyzed were de-identified, as required under the US Health Insurance Portability and Accountability Act privacy rule.

Data Availability. The data used in this study were derived from US insurance claims data from the IQVIA ${ }^{\mathrm{TM}}$ RWD Adjudicated Claims-US database. Access to these data is available upon request.

Open Access. This article is distributed under the terms of the Creative Commons Attribution-NonCommercial 4.0 International License (http://creativecommons.org/licenses/ by-nc/4.0/), which permits any noncommercial use, distribution, and reproduction in any medium, provided you give appropriate credit to the original author(s) and the source, provide a link to the Creative Commons license, and indicate if changes were made. 


\section{REFERENCES}

1. Ananthakrishnan AN. Epidemiology and risk factors for IBD. Nat Rev Gastroenterol Hepatol. 2015;12:205-17.

2. Dahlhamer JM, Zammitti EP, Ward BW, et al. Prevalence of inflammatory bowel disease among adults aged $\geq 18$ years-United States, 2015. Morb Mortal Wkly Rep. 2016;65:1166-9.

3. Kaplan GG. The global burden of IBD: from 2015 to 2025. Nat Rev Gastroenterol Hepatol. 2015;12:720-7.

4. Mehta F. Economic implications of inflammatory bowel disease and its management. Am J Manag Care. 2016;22:S51-60.

5. Floyd DN, Langham S, Severac HC, et al. The economic and quality-of-life burden of Crohn's disease in Europe and the United States, 2000-2013: a systematic review. Dig Dis Sci. 2015;60:299-312.

6. Limsrivilai J, Stidham RW, Govani SM, et al. Factors that predict high health care utilization and costs for patients with inflammatory bowel diseases. Clin Gastroenterol Hepatol. 2017;15:385-92.

7. Sandborn W, Binion D, Persley K, Atreja A, Kosinski L. AGA Institute guidelines for the identification, assessment and initial medical treatment in Crohn's disease: clinical decision support tool. American Gastroenterological Association. https://s3. amazonaws.com/agaassets/pdf/guidelines/IBDCare Pathway.pdf. Accessed 31 Jan 2019.

8. American Gastroenterological Association. Guidelines for the identification, assessment and initial medical treatment of ulcerative colitis, 2015. https://s3.amazonaws.com/agaassets/pdf/guidelines/ UlcerativeColitis/index.html. Accessed 31 Jan 2019.

9. World Gastroenterology Organisation. World Gastroenterology Organisation global guidelines: inflammatory bowel disease. Updated August 2015. http://www.worldgastroenterology.org/guidelines/ global-guidelines/inflammatory-bowel-disease-ibd/ inflammatory-bowel-disease-ibd-english. Accessed 31 Jan 2019.

10. Gomollon F, Dignass A, Annese V, et al. 3rd European evidence-based consensus on the diagnosis and management of Crohn's disease 2016: part 1: diagnosis and medical management. J Crohn's Colitis. 2016;2017(11):3-25.

11. Harbord M, Eliakim R, Bettenworth D, et al. 3rd European evidence-based consensus on diagnosis and management of ulcerative colitis. Part 2: current management. J Crohn's Colitis 2017. (Epub prior to print).

12. Lichtenstein GR, Hanauer SB, Sandborn WJ. Management of Crohn's disease in adults. Am J Gastroenterol. 2009;104:465-83.

13. Mowat C, Cole A, Windsor A, et al. Guidelines for the management of inflammatory bowel disease in adults. Gut. 2011;60:571-607.

14. Wehkamp J, Gotz M, Herrlinger K, et al. Inflammatory bowel disease: Crohn's disease and ulcerative colitis. Dtsch Arztebl Int. 2016;113:72-82.

15. Rowe WA. Inflammatory bowel disease treatment and management, 2016.

16. Mao R, Hu PJ. The future of IBD therapy: where are we and where should we go next? Dig Dis. 2016;34:175-9.

17. Peyrin-Biroulet L, Sandborn W, Sands BE, et al. Selecting therapeutic targets in inflammatory bowel disease (STRIDE): determining therapeutic goals for treat-to-target. Am J Gastroenterol. 2015;110:1324-38.

18. McLean LP, Cross RK. Adverse events in IBD: to stop or continue immune suppressant and biologic treatment. Expert Rev Gastroenterol Hepatol. 2014;8:223-40.

19. Marehbian J, Arrighi HM, Hass S, et al. Adverse events associated with common therapy regimens for moderate-to-severe Crohn's disease. Am J Gastroenterol. 2009;104:2524-33.

20. McAuliffe ME, Lanes S, Leach T, et al. Occurrence of adverse events among patients with inflammatory bowel disease in the HealthCore Integrated Research Database. Curr Med Res Opin. $2015 ; 31: 1655-64$.

21. Kappelman MD, Porter CQ, Galanko JA, et al. Utilization of healthcare resources by US children and adults with inflammatory bowel disease. Inflamm Bowel Dis. 2011;17:62-8.

22. Bassi A, Dodd S, Williamson P, et al. Cost of illness of inflammatory bowel disease in the UK: a single centre retrospective study. Gut. 2004;53:1471-8.

23. Bickston SJ, Waters HC, Dabbous O, et al. Administrative claims analysis of all-cause annual costs of care and resource utilization by age category for ulcerative colitis patients. J Manag Care Pharm. 2008;14:352-62.

24. Peery AF, Crockett SD, Barritt AS, et al. Burden of gastrointestinal, liver, and pancreatic diseases in 
the United States. Gastroenterology. 2015;149:1731-41.

25. van Deen $W K$, van Oijen MG, Myers $K D$, et al. A nationwide 2010-2012 analysis of US health care utilization in inflammatory bowel diseases. Inflamm Bowel Dis. 2014;20:1747-53.

26. Lewis JD, Aberra FN, Lichtenstein GR, et al. Seasonal variation in flares of inflammatory bowel disease. Gastroenterology. 2004;126:665-73.

27. Liu X, Kubilis P, Bussing R, et al. Development of a refill pattern method to measure polypharmacy in administrative claims databases. Pharmacoepidemiol Drug Saf. 2016;25:1407-13.

28. Bahler C, Schoepfer AM, Vavricka SR, et al. Chronic comorbidities associated with inflammatory bowel disease: prevalence and impact on healthcare costs in Switzerland. Eur J Gastroenterol Hepatol. 2017;29:916-25.

29. Abegunde AT, Muhammad BH, Ali T. Preventive health measures in inflammatory bowel disease. World J Gastroenterol. 2016;22:7625-44.

30. Charlson M, Szatrowski TP, Peterson J, et al. Validation of a combined comorbidity index. J Clin Epidemiol. 1994;47:1245-51.

31. Ananthakrishnan AN, McGinley EL, Binion DG, et al. A novel risk score to stratify severity of
Crohn's disease hospitalizations. Am J Gastroenterol. 2010;105:1799-807.

32. Peduzzi P, Concato J, Feinstein AR, et al. Importance of events per independent variable in proportional hazards regression analysis. II. Accuracy and precision of regression estimates. J Clin Epidemiol. 1995;48:1503-10.

33. Concato J, Peduzzi P, Holford TR, et al. Importance of events per independent variable in proportional hazards analysis. I. Background, goals, and general strategy. J Clin Epidemiol. 1995;48:1495-501.

34. Buchman AL. Side effects of corticosteroid therapy. J Clin Gastroenterol. 2001;33:289-94.

35. Roblin X, Danese S, Peyrin-Biroulet L. Does antiTNF therapy costs so many COINs? Gastroenterology. 2014;146:309-11.

36. Sondag M, Verhoeven F, Guillot X, et al. "Paradoxical" arthralgia occurring under anti-TNFalpha treatment for inflammatory bowel disease. Jt Bone Spine. 2018;85:133-4.

37. Wendling D. The gut in spondyloarthritis. Jt Bone Spine. 2016;83:401-5.

38. Huoponen S, Blom M. A systematic review of the cost-effectiveness of biologics for the treatment of inflammatory bowel diseases. PLOS ONE. 2015;10:e0145087. 